\title{
Analyse graphique de mesures d'électrogravimétrie en régime dynamique pour des films minces électroactifs
}

\author{
D. Giménez-Romero1, P.R. Bueno², C. Gabrielli³, J. García-Jareño', \\ H. Perrot ${ }^{3}$ et F. Vicente ${ }^{1}$ \\ 1 Departament Química Física. /Dr. Moliner, 50, 46100 Burjassot, València, Espagne \\ e-mail : giroda@uv.es; Jose.J.Garcia@uv.es; Francisco.Vicente@uv.es \\ 2 Instituto de Química, Departamento de Físico-Química, Universidade Estadual Paulista, PO Box 355, \\ 14801-907, Araraquara, São Paulo, Brésil \\ e-mail : prbueno@mail.iq.unesp.br \\ 3 Université P. et M. Curie, UPR 15 du CNRS-LISE, 4 place Jussieu, 75252 Paris, France \\ e-mail : claude.gabrielli@upmc.fr; hubert.perrot@upmc.fr
}

\section{Mots-clés :}

Électrogravimétrie; films électroactifs; points singuliers

\section{Key words:}

Electrogravimetry; electroactive films; singular points
Résumé - Différentes fonctions de transfert caractéristiques d'un système électrochimique, associé à des films électroactifs minces, peuvent être calculées. En général, les processus de compensation de charges couplés à la cinétique hétérogène sont à la base des différentes théories proposées. Les paramètres cinétiques, associés au mécanisme de réaction, peuvent être calculés directement à partir des points singuliers associés à différents diagrammes et différentes fonctions de transfert (Cole-Cole, fonction $F \frac{\Delta m}{\Delta q} \ldots$ ). En conséquence, une stratégie pour estimer ces paramètres cinétiques est proposée dans ce travail et analysée sur un organigramme détaillé. Les fonctions de transfert obtenues peuvent aussi permettre une corrélation entre l'aspect cinétique et la structure des films étudiés.

\begin{abstract}
Graphical analysis of electrogravimetric measurements under dynamic regime applied to electroactive films. Various transfer functions associated to an electrochemical process can be calculated for thin electroactive films. In general, the theory is based on a charge compensation reaction coupled with heterogeneous kinetic. The kinetic parameters can be estimated using different approaches. In this paper, a direct estimation, based on singular points, is proposed by using different transfer functions as Cole-cole or $F \frac{\Delta m}{\Delta q}$ transfer functions. Therefore, a strategy is proposed to estimate these parameters and a detailed diagram is given. Moreover, a correlation is established between the kinetic aspect and the structural properties of the films examined.
\end{abstract}

a qualité des films électroactifs, type électrochrome ou films utilisés pour les batteries, est corrélée à la facilité de transfert d'espèces chargées au niveau des interfaces métal/film et film/électrolyte et à la mobilité de ces espèces dans le film mince compris entre ces deux interfaces [1-7]. La Spectroscopie d'Impédance Électrochimique (SIE) est une des techniques les plus utilisé pour caractériser les propriétés de ces systèmes électrochimiques [8-10]. L'analyse traditionnelle des mesures obtenues avec ces techniques de courant alternatif consiste à ajuster des fonctions de transferts théoriques, déduites de modèles de type circuit équivalent ou cinétique, aux résultats expérimentaux. Des méthodes d'ajustement non linéaires sont alors employées [12-14] et en conséquence, des programmes spécialisés sont nécessaire [12,13]. Toutefois, d'autres méthodes d'analyse sont possibles.

Actuellement, l'analyse graphique de la fonction d'immittance peut être appliquée en terme qualitatif et quantitatif [15-17]. L'avantage principal est la possibilité d'identifier et d'estimer rapidement les différentes constantes de temps, d'analyser le comportement dispersif... [18]. Dans ce travail, différentes simulations de diagrammes d'immittances sont proposées et analysées en terme de points singuliers. 


\section{Approche théorique}

Dans notre cas, le mécanisme d'insertion/expulsion correspondant à l'interaction d'espèces chargées en solution avec un film électroactif peut s'écrire pour un cation :

$$
\left\langle H, C_{i}^{x+}\right\rangle \underset{k_{\text {red }}}{\stackrel{k_{\text {oxi }}}{\rightleftarrows}}\langle H\rangle+z e^{-}+\frac{z}{x} C_{i}^{x+}
$$

et pour un anion :

$$
\langle H\rangle+\frac{z}{x} A_{i}^{x-} \underset{k_{\text {red }}}{\stackrel{k_{\text {oxi }}}{\rightleftarrows}}\left\langle H, A_{i}^{x-}\right\rangle+z e^{-} .
$$

L'impédance électrochimique, $Z_{i}(\omega)$, de ces systèmes repose sur l'addition d'une contribution Faradique, $Z_{f}(\omega)$, et une partie nonFaradique. L'impédance électrochimique se compose d'une capacité de double couche électrique, $C_{d l}$, parallèlement à l'impédance Faradique, $Z_{f}(\omega)$, l'ensemble étant en série avec la résistance d'électrolyte, $R_{u}$. Ces paramètres non-Faradiques peuvent être facilement calculés à partir des valeurs de l'impédance expérimentale à hautes fréquences [3] L'impédance électrochimique vaut alors :

$$
Z_{i}(\omega)=\frac{Z_{f}(\omega)}{1+j \omega C_{d l} Z_{f}(\omega)}+R_{u} .
$$

Comme la contribution non-Faradique peut être facilement calculée, nous étudierons uniquement dans ce papier l'impédance Faradique. La fonction de transfert charge/potentiel, $C(\omega)$, correspondant à ce mécanisme de réaction, a déjà été calculée pour des films minces en négligeant les phénomènes de diffusions c'est-à-dire si $1 / \omega_{d i} \gg$ $L^{2} / D_{i}[3,4]$ où $\omega_{d i}$ est la fréquence caractéristique pour un processus de diffusion $i, D_{i}$ étant la constant de diffusion et $L$ l'épaisseur du film électroactif :

$$
C(\omega)=\frac{\Delta \sum_{i=1}^{n} q_{i}(\omega)}{\Delta E(\omega)}=\sum_{i=1}^{n} z_{i} V_{h} F \frac{-G_{i}}{j \omega+K_{i}}
$$

où

$$
\begin{gathered}
G_{i}=b_{\text {red }} k_{\text {red }}^{0}\left(N_{j}-\eta_{i}\right)-b_{\text {oxi }} k_{\text {oxi }}^{0} \eta_{i} \\
K_{i}=k_{\text {red }}^{0}+k_{\text {oxi }}^{0} .
\end{gathered}
$$

La distribution des sites " électroactifs » au sein de ces films est décrite en terme de la fraction d'occupation, $\eta_{i} / N_{j}$, où $\eta_{i}$ est la densité du nombre d'atomes intercalés pour l'espèce $i$ et $N_{j}$ est la densité du nombre d'emplacement de type $j$. Il est à noter que $\left(N_{j}-\eta_{i}\right)$ est la densité du nombre d'emplacements disponibles pour l'intercalation de cations ou la densité du nombre d'anions intercalés, $\eta_{i}$ est la densité du nombre des cations intercalés ou des emplacements disponibles pour l'intercalation des anions, $z_{i}$, est la charge élémentaire de l'ion impliqué, $F$ le nombre de faraday et $V_{h}$ le volume du film électroactif. $k_{\text {red }}^{0}$ et $k_{o x i}^{0}$ sont, respectivement, les constantes cinétiques des processus de réduction et d'oxydation. Ces derniers paramètres dépendent exponentiellement du potentiel [19], $k_{i}^{0}=\overline{k_{i}} \exp \left(b_{i} \bar{V}\right)$ où $b_{\text {red }}$ et $b_{\text {oxi }}$ sont les facteurs pré-exponentiels. Les processus cinétiques irréversibles peuvent être facilement décrits : d'une part, si $k_{\text {red }} / k_{\text {oxi }} \rightarrow \infty$ $\left(k_{\text {oxi }}<<k_{\text {red }}\right)$ alors $G_{i}=b_{\text {red }} k_{\text {red }}^{0}\left(N_{y}-\overline{\eta_{i}}\right)$ et d'autre part, si $k_{\text {red }} / k_{\text {oxi }} \rightarrow 0\left(k_{\text {oxi }}>>k_{\text {red }}\right)$ alors $G_{i}=-b_{\text {oxi }} k_{\text {oxi }}^{0} \bar{\eta}_{i}$.

\section{Analyse graphique des dia- grammes d'immittance faradique}

La connaissance de la fonction de transfert théorique $C(\omega)$ dans le domaine fréquentiel nous permet de faire une analyse des diagrammes d'immittances associés à l'électrogravimétrie. Basé sur les conditions aux limites, l'analyse graphique de ces diagrammes d'immittances fournit une méthodologie facile pour obtenir les paramètres cinétiques.

Le diagramme de Nyquist de l'impédance Faradique, $Z_{f}(\omega)$, est calculé théoriquement grâce à l'équation :

$$
Z_{f}(\omega)=\frac{1}{j \omega C(\omega)}=Z^{\prime}(\omega)+Z^{\prime \prime}(\omega) j .
$$

Ce diagramme est la représentation de la partie imaginaire, $Z^{\prime \prime}(\omega)$, en fonction de la partie réelle, $Z^{\prime}(\omega)$, de cette fonction complexe d'impédance Faradique (Fig. 1). Comme cette figure le montre, la fonction théorique présente deux limites caractéristiques pour des fréquences qui tendent à l'infini (1st) et vers zéro (2nd). Ainsi, nous obtenons :

$$
\begin{gathered}
Z_{\omega \rightarrow \infty}=\frac{1}{F V_{h} \sum_{i=1}^{n}-z_{i} G_{i}} \\
Z_{\omega \rightarrow 0}^{\prime}=\frac{\sum_{i=1}^{n}-z_{i} \frac{G_{i}}{K_{i}^{2}}}{F V_{h}\left(\sum_{i=1}^{n}-z_{i} \frac{G_{i}}{K_{i}}\right)^{2}}
\end{gathered}
$$




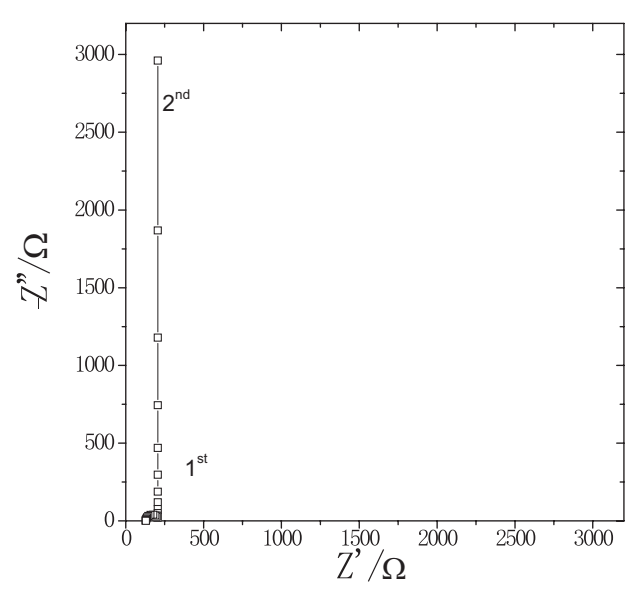

Fig. 1. Diagramme de Nyquist de l'impédance électrochimique, $Z(\omega)$, basé sur la fonction indiquée dans l'équation (2). Les paramètres cinétiques de cette simulation sont: $G_{1}=-0,05 \mathrm{~mol}$ $\mathrm{V}^{-1} \mathrm{~s}^{-1} \mathrm{~cm}^{-3}, K_{1}=0,9 \mathrm{~s}^{-1}, G_{2}=-0,03 \mathrm{~mol} \mathrm{~V}^{-1}$ $\mathrm{s}^{-1} \mathrm{~cm}^{-3}, K_{2}=200 \mathrm{~s}^{-1}$ et $V_{h}=3,810^{-6} \mathrm{~cm}^{3}$.

Fig. 1. Nyquist plot of the electrochemical impedance, $Z(\omega)$, calculated from equation (2). Kinetic parameters are: $G_{1}=-0.05 \mathrm{~mol} \mathrm{~V}-1 \mathrm{~s}^{-1} \mathrm{~cm}^{-3}, K_{1}=0.9 \mathrm{~s}^{-1}, G_{2}=$ $-0.03 \mathrm{~mol} \mathrm{~V} \mathrm{~V}^{-1} \mathrm{~s}^{-1} \mathrm{~cm}^{-3}, K_{2}=200 \mathrm{~s}^{-1}$ and $V_{h}=3.8$ $10^{-6} \mathrm{~cm}^{3}$.

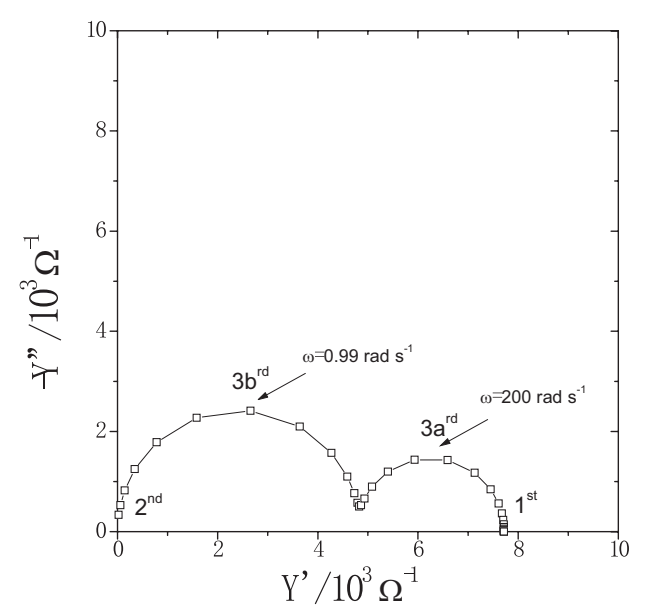

Fig. 2. Diagramme d'admittance, $Y(\omega)$. Les paramètres cinétiques de cette simulation sont décrits dans la figure 1

Fig. 2. Electrochemical admittance, $Y(\omega)$. Kinetic parameters are given in Figure 1.

$$
Z_{\omega \rightarrow 0}^{\prime \prime}=\infty .
$$

En conséquence, la partie réelle de l'impédance Faradique en zéro, $Z_{\omega \rightarrow 0}^{\prime}$, est inversement proportionnelle au volume du film et donc à l'épaisseur de ce dernier en connaissant la surface de dépôt.

Le diagramme d'admittance, $Y(\omega)$, peut aussi être fait directement en utilisant l'équation suivante (Fig. 2).

$$
Y(\omega)=j \omega C(\omega)
$$

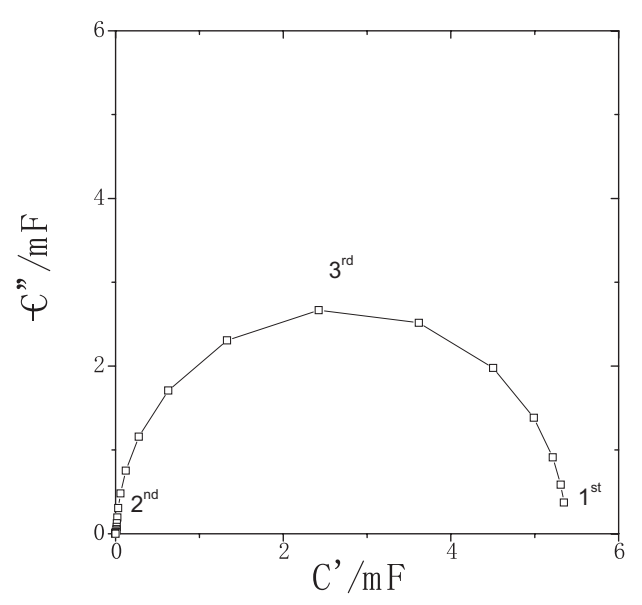

Fig. 3. Diagramme complexe, $C(\omega)$, de la fonction capacité. Les paramètres cinétiques de cette simulation sont décrits dans la figure 1.

Fig. 3. Complex diagram of the capacitance, $C(\omega)$. Same parameters as in Figure 1.

$$
\begin{gathered}
Y(\omega)=j \omega \sum_{i=1}^{n} z_{i} V_{h} F \frac{-G_{i}}{j \omega+K_{i}} \\
Y(\omega)=\sum_{i=1}^{n} z_{i} V_{h} F \frac{-G_{i}}{\frac{j \omega}{j \omega}+\frac{K_{i}}{j \omega}} \\
Y(\omega)=\sum_{i=1}^{n} z_{i} V_{h} F \frac{G_{i}}{\left(j K_{i} / \omega\right)-1} .
\end{gathered}
$$

La figure 2 montre trois limites caractéristiques : fréquence tendant à l'infini (1st), à zéro (2nd) et quand la partie imaginaire de la fonction d'admittance a une valeur minimum (3rd). Le premier point est :

$$
Y_{\omega \rightarrow \infty}=F V_{h} \sum_{i=1}^{n}-z_{i} G_{i}
$$

Le diagramme capacitif est la représentation de la partie imaginaire, $C^{\prime \prime}(\omega)$, en fonction de la partie réelle, $C^{\prime}(\omega)$, de la fonction complexe de la capacité, $C(\omega)$ (Fig. 3) :

$$
\begin{aligned}
C(\omega) & =\sum_{i=1}^{n} z_{i} V_{h} F \frac{-G_{i} K_{i}}{K_{i}^{2}+\omega^{2}}+j \sum_{i=1}^{n} z_{i} V_{h} F \frac{\omega G_{i}}{K_{i}^{2}+\omega^{2}} \\
& =C^{\prime}(\omega)+j C^{\prime \prime}(\omega) .
\end{aligned}
$$

Comme la figure 3 le montre, cette fonction théorique présente également trois points caractéristiques : quand la fréquence tendant à l'infini (1st), à zéro (2nd) et quand la partie imaginaire de la fonction de capacité a une valeur minimum maximum (3rd). Ce deuxième point caractéristique vaut :

$$
C_{\omega \rightarrow 0}=F V_{h} \sum_{i=1}^{n} z_{i} \frac{-G_{i}}{K_{i}} .
$$




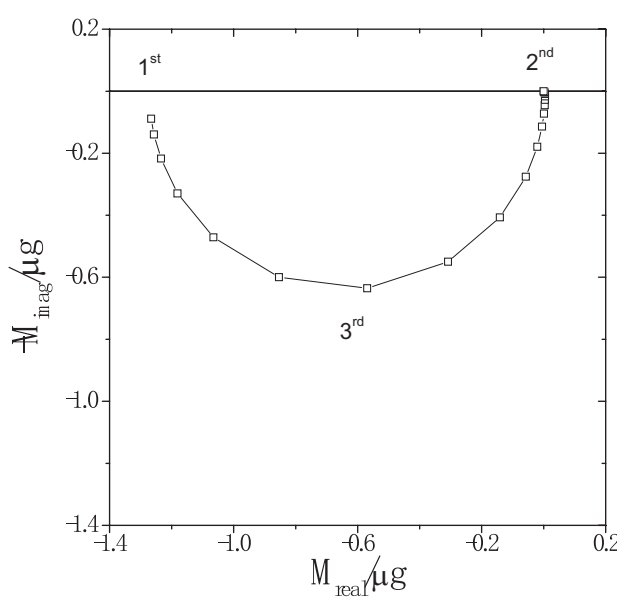

Fig. 4. Diagramme masse-potentiel. Les paramètres cinétiques de cette simulation sont les mêmes que ceux de la figure 1. Dans ce diagramme, $M W_{1}$ (cation) $=23 \mathrm{~g} \mathrm{~mol}^{-1}$ et $M W_{2}$ (anion) $=35 \mathrm{~g} \mathrm{~mol}^{-1}$.

Fig. 4. Mass-potential diagram. Same parameters as in Figure 1. In this diagram, $M W_{1}$ (cation) $=23 \mathrm{~g} \mathrm{~mol}^{-1}$ and $\mathrm{MW}_{2}$ (anion) $=35 \mathrm{~g} \mathrm{~mol}^{-1}$.

Ce point caractéristique dépend directement des emplacements d'anions ou de cations avec la plus grande densité structurale, $N_{j}$, puisque :

$$
\frac{G_{i}}{K_{i}}=\frac{b_{r e d} k_{r e d}^{0}\left(N_{j}-\eta_{i}\right)-b_{o x i} k_{o x i}^{0} \eta_{i}}{k_{r e d}^{0}+k_{o x i}^{0}} \approx 2 b N_{j} .
$$

En plus, la capacité à cette fréquence est une estimation directe de l'épaisseur du film, $L$.

La représentation (Fig. 4) de la fonction de transfert masse-potentiel, $d m / d V$, est obtenue à partir de l'équation (18). Il est très important de discuter les points caractéristiques en considérant un échange de cations et/ou d'anions car le modèle peut changer. L'aspect du diagramme $d m / d V$ est très semblable à celui du diagramme capacitif, $C(\omega)$, si seulement des cations ou des anions sont impliqués. Ceci se produit parce que la masse est quasiment proportionnelle à la densité de courant :

$$
\begin{array}{r}
\frac{d m}{d V}(\omega)=F \sum_{i=1}^{n} \alpha_{i} V_{h} \frac{z_{i}}{x_{i}} M W_{i} \frac{-G_{i}}{j \omega+K_{i}} \\
F \frac{d m}{d Q}(\omega)=\frac{\sum_{i=1}^{n} \alpha_{i} \frac{z_{i}}{x_{i}} M W_{i} \frac{-G_{i}}{j \omega+K_{i}}}{\sum_{i=1}^{n} z_{i} \frac{-G_{i}}{j \omega+K_{i}}}
\end{array}
$$

où $M W_{i}$ est la masse molaire, $x_{i}$ est la charge des espèces intercalées et $z_{i}$ est le nombre d'électrons intercalés par centre actif. $\alpha_{i}$ est supposé être -1 quand il y a un processus cinétique impliquant des espèces cationiques et +1 quand il y a un processus cinétique uniquement avec des espèces anioniques.

Il est important de noter que la localisation des boucles relatives aux cations et anions est dans des quadrants opposés. Par conséquent, en analysant la dernière équation correspondant à la fonction de transfert masse-potentiel il vient : une valeur négative (positive) de la partie réelle et une valeur positive (négative) de sa partie imaginaire quand les espèces échangées avec le centre récepteur sont principalement des cations (anions). Les valeurs intermédiaires indiquent l'échange simultané des anions et des cations. Par conséquent, ce diagramme permet de discerner facilement le mouvement des cations et/ou des anions pendant les processus électrochimique si les constantes de temps sont suffisamment séparées.

Après ces quelques commentaires, les points caractéristiques liés à cette fonction vont être donnés. La fonction théorique présente trois limites caractéristiques : fréquence tendant à l'infini (1st), vers zéro (2nd) et les valeurs maximum et minimum de la partie imaginaire de la fonction (3rd). La deuxième limite caractéristique correspond à :

$$
\left.\frac{d m}{d V}\right|_{\omega \rightarrow 0}=\sum_{i=1}^{n} \alpha_{i} F V_{h} \frac{z_{i}}{x_{i}} M W_{i} \frac{-G_{i}}{K_{i}} .
$$

Cette limite caractéristique peut être une bonne évaluation des masses molaires des espèces échangées pendant les transferts ioniques. Sous certaines conditions, il vient :

$$
\left.\left.\frac{d m}{d V}\right|_{\omega \rightarrow 0} \approx \alpha_{i} F V_{h} \frac{z_{i}}{x_{i}} M W_{i} \frac{-G_{i}}{K_{i}}\right|_{\frac{G_{i}}{K_{i}}>>\text { autres_reactions }}
$$

$M W_{i}$ peut être calculée facilement en combinant cette limite caractéristique et la valeur de $C_{\omega \rightarrow 0}$, (Éq. (16)).

Le diagramme de la fonction de transfert mass/charge électrique (diagramme $F(d m / d Q)$ ) est la représentation de la partie imaginaire de la fonction $F(d m / d Q)$ (Éq. (19)) en fonction de la partie réelle (Fig. 5). Cette fonction est constante si seulement une espèce est impliquée dans le processus électrochimique ou deux espèces de même charges avec des constantes cinétiques très proches. La fonction $F(d m / d Q)$ présente trois points caractéristiques : fréquence tendant à l'infini (1st), à la valeur zéro (2nd) et aux valeurs maximum et minimum de la partie imaginaire de la fonction (3rd). La deuxième limite 


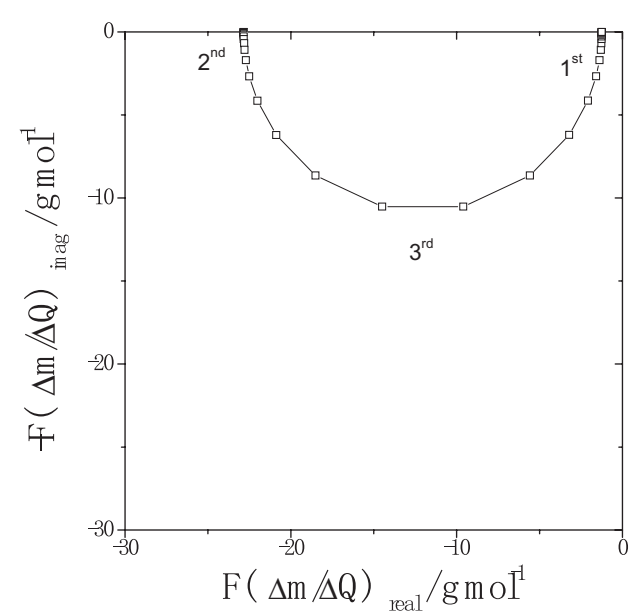

Fig. 5. Diagramme complexe de la fonction $F(d m / d Q)$. Les paramètres cinétiques de cette simulation sont les mêmes que ceux de la figure 1 . $M W_{1}($ cation $)=23 \mathrm{~g} \mathrm{~mol}^{-1}$ et $M W_{2}($ anion $)=35$ $\mathrm{g} \mathrm{mol}^{\mathbf{- 1}}$.

Fig. 5. Complex diagram of the function $F(d m / d Q)$. Same parameters as in Figure 1. $M W_{1}$ (cation) = $23 \mathrm{~g} \mathrm{~mol}^{-1}$ and $\mathrm{MW}_{2}$ (anion) $=35 \mathrm{~g} \mathrm{~mol}^{-1}$.

caractéristique dépend principalement de $G_{i} / K_{i}$ :

$$
\left.F \frac{d m}{d Q}\right|_{\omega \rightarrow 0}=\frac{\sum_{i=1}^{n} \alpha_{i} \frac{z_{i}}{x_{i}} M W_{i} \frac{G_{i}}{K_{i}}}{\sum_{i=1}^{n} z_{i} \frac{G_{i}}{K_{i}}} .
$$

Les analyses proposées ici ont été conduites principalement en explorant les aspects graphiques des diagrammes pour en extraire les limites des fonctions complexes. Cette approche relativement simple permet de s'affranchir des étapes de type simulation ou ajustement avec des fonctions complexes. Cela permet d'accéder facilement aux grandeurs intéressantes caractéristiques du processus comme les constantes cinétiques d'insertion ou les masses molaires car il est possible d'établir un système d'équations à partir de ces points.

\section{Remerciements}

Ce travail a été soutenu par le projet CTQ 2004-08026/BQU de FEDER-CICyT.
D. Giménez-Romero a reçu une aide de la Generalitat Valenciana et Paulo R. Bueno doit sa position à l'organisme de recherches de l'état de São Paulo (FAPESP) sous le projet numéro 02/06693-3.

\section{Références}

[1] C. Gabrielli, J.J. Garci-Jareño, H. Perrot, Electrochim. Acta 46 (2001) 4095

[2] C. Gabrielli, J.J. Garcia-Jareño, M. Keddam, H. Perrot, F. Vicente, J. Phys. Chem. B 106 (2002) 3182

[3] D. Gimenez-Romero, P.R. Bueno, C. Gabrielli, H. Perrot, J.J. Garcia-Jareño, F. Vicente, J. Phys. Chem. 110 (2006) 19352

[4] D. Gimenez-Romero, P.R. Bueno, C. Gabrielli, H. Perrot, J.J. Garcia-Jareño, F. Vicente, J. Phys. Chem. 110 (2006) 19364

[5] C. Gabrielli, J.J. Garcia-Jareño, M. Keddam, H. Perrot, F. Vicente, J. Phys. Chem. B 106 (2002) 3192

[6] G. Inzelt, E. Csahok, Electroanal. 11 (1999) 744

[7] A. Neudeck, A. Petr, L. Dunsch, Synth. Metals 107 (1999) 143

[8] M.A. Vorotyntsev, L.I. Dainkin, M.D. Levi, J. Electroanal. Chem. 364 (1994) 37

[9] C. Deslouis, M. Musiani, B. Tribollet, J. Phys. Chem. 100 (1996) 8994

[10] G. Lang, G. Inzelt, Electrochim. Acta 44 (1999) 2037

[11] M.F. Mathias, O. Hass, J. Phys. Chem. 96 (1992) 3174

[12] J.R. Macdonald, Solid State Ionics 58 (1992) 97

[13] B.A. Boukamp, Solid State Ionics 16 (1986) 136

[14] R.H. Milocco, J. Electroanal. Chem. 279 (1989) 243

[15] D. Giménez-Romero, J.J. García-Jareño, F. Vicente, Electrochem. Comm. 5 (2003) 722

[16] I. Rubinstein, E. Sabatani, J. Rishpon, J. Electrochem. Soc. 3078 (1987) 134

[17] Z. Stoynov, Pol. J. Chem. 71 (1997) 1204

[18] D. Vladikova, P. Zoltowski, E. Makowska, Z. Stoynov, Electrochim. Acta 47 (2002) 2943

[19] L. Bortels, B. Van den Bossche, J. Deconinck, S, Vandeputte, A. Hubin, J. Electroanal. Chem. 429 (1997) 139 